\title{
RECURRENCE OF POSTERIOR REVERSIBLE ENCEPHALOPATHY SYNDROME (PRES) AS A SEVERE NEUROLOGICAL MANIFESTATION OF SYSTEMIC LUPUS ERYTHEMATOSUS (SLE) - CASE REPORT
}

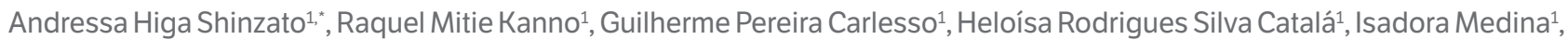
Marcela Miguens Castelar Pinheiro ${ }^{1}$, Stella Falcadi Vendramine ${ }^{1}$, Felipe de Lacerda Veiga ${ }^{1}$, Pedro Matos ${ }^{1}$, Edgard Torres dos Reis Neto ${ }^{1}$

1. Universidade Federal de São Paulo, São Paulo (SP), Brazil.

*Corresponding author: dessa_higa@hotmail.com

\section{BACKGROUND}

Posterior reversible encephalopathy (PRES) is a syndrome characterized by seizures, hypertension, headache, disorders of consciousness and focal neurologic deficits. Its pathophysiology is not well understood and one of the proposed mechanisms is a failure of cerebrovascular autoregulation and hyperperfusion, and also an endothelial lesion due to some risk factors as hypertension, uremia, systemic lupus erythematosus (SLE) and some drugs, as cyclophosphamide.

\section{CASE REPORT}

A 32-year-old female with SLE juvenile onset was hospitalized due to lupus nephritis (LN) class IV/V treated with $1 \mathrm{~g}$ of intravenous methylprednisolone for 3 days followed by oral prednisone ( $1 \mathrm{mg} / \mathrm{kg} /$ day), hydroxychloroquine and $1 \mathrm{~g}$ of intravenous cyclophosphamide $(0.7 \mathrm{~g} / \mathrm{m} 2$ of body surface area). After 3 days, she presented seizures and hypertension. Magnetic resonance imaging (MRI) of the brain showed white matter hyperintensities in both cerebral hemispheres affecting the frontal, parietal and left occipital lobes (Fig. 1). Diagnosis of PRES associated with the recent use of cyclophosphamide or due to SLE activity was performed. The patient was discharged from hospital with mycophenolate as induction therapy for LN-associated antiepileptic and antihypertensive drugs. Another MRI performed after 23 days of treatment revealed completed resolution of the brain lesions (Fig. 2). After 5 days, she returned to the emergency with new episodes of seizures, acute renal failure (ARF) and hypertension. The new MRI demonstrated new lesions suggestive of PRES (Fig. 3). After 3 weeks treating with corticosteroids in immunosuppressive dose, the patient was discharged with no neurological deficit. Four months later, she presented diarrhea, fever, pancytopenia and ARF. A fourth MRI demonstrated few areas associated with PRES (Fig. 4). The patient was diagnosed with disseminated cryptococcosis by chest tomography and detection of capsular polysaccharide antigen of Cryptococcus neoformans. Unfortunately, the patient did not respond to antifungal therapy and died.

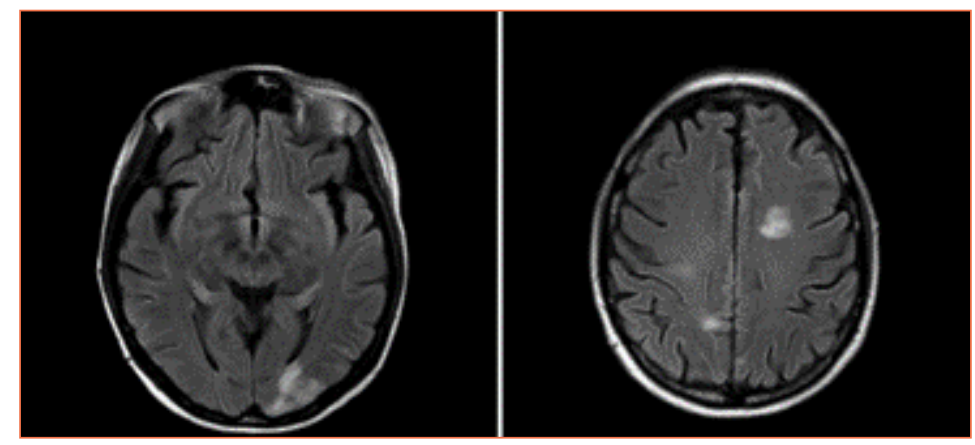

Figure 1. White matter hyperintensities in both cerebral hemispheres. 


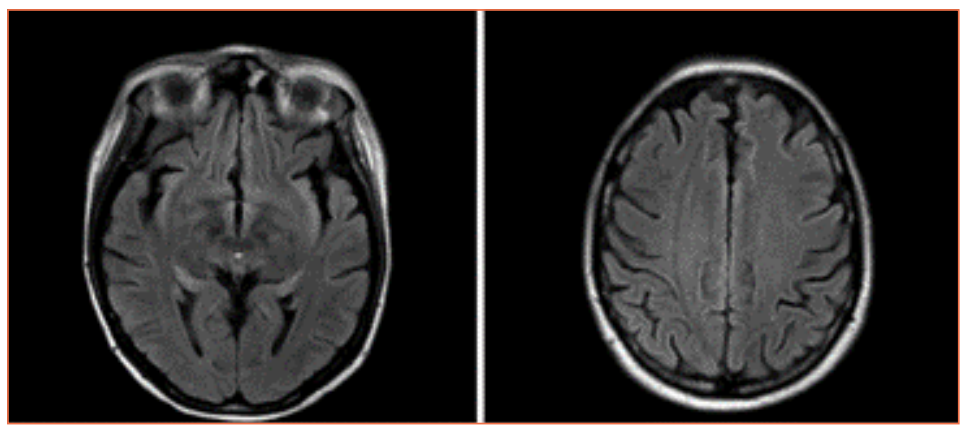

Figure 2. Resolution of areas in new MRI images.

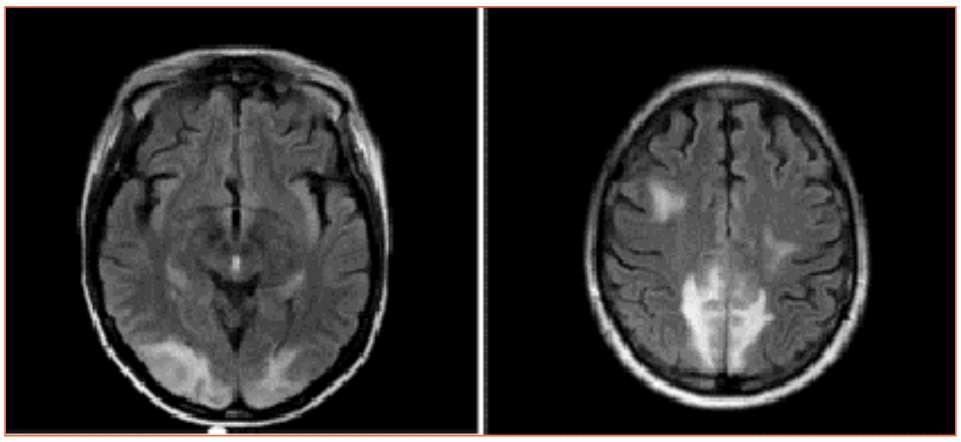

Figure 3. Appearance of new areas with hypersignal in the third MRI.
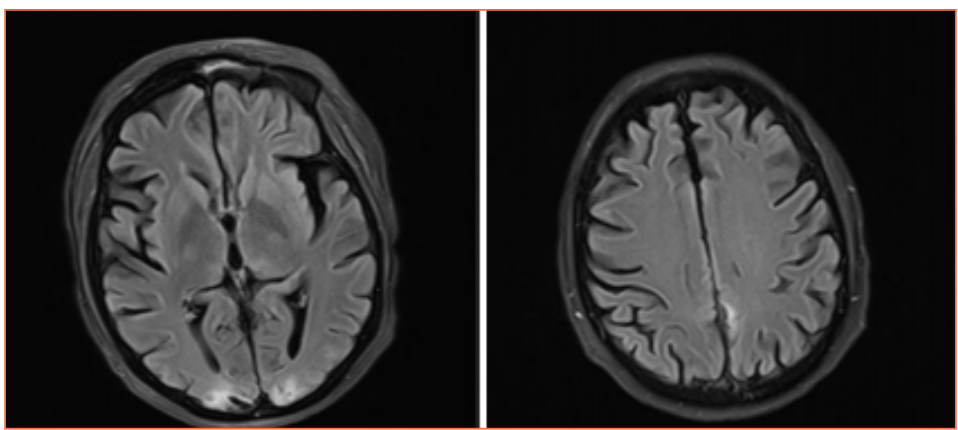

Figure 4. Reduction of hypersignal in the bilateral parieto-occipital and frontal regions.

\section{CONCLUSION}

This case report describes the recurrence of PRES despite treatment in a SLE patient with severe manifestations. Although cyclophosphamide has been described as a drug associated of PRES, neurological SLE activity must be appropriately treated to prevent damage and recurrence. 